\title{
Mesenchymal stem cells and exosome therapy for COVID-19: current status and future perspective
}

\author{
Ashim Gupta ${ }^{1,2,3,4} \cdot$ Shivaji Kashte ${ }^{5} \cdot$ Manu Gupta ${ }^{1}$ Hugo C. Rodriguez ${ }^{1,3,6} \cdot$ Shraddha Singh Gautam ${ }^{7}$. \\ Sachin Kadam ${ }^{5,7}$
}

Received: 20 July 2020 / Accepted: 28 July 2020 / Published online: 11 August 2020

(c) Japan Human Cell Society 2020

\begin{abstract}
Acute respiratory distress syndrome (ARDS) is the main cause for the COVID-19 infection-related morbidity and mortality. Recent clinical evidences suggest increased level of cytokines and chemokines targeting lung tissue as a prominent etiological factor. The immunomodulatory effect of mesenchymal stem cells (MSCs) as the alternative therapy for the treatment of inflammatory and autoimmune diseases is well known. Several studies have also revealed that similar therapeutic impacts of parent MSCs are also exhibited by MSCs-derived extracellular vesicles (EVs) including exosomes. In this review, we explored the therapeutic potential of both MSCs and exosomes in mitigating the COVID-19 induced cytokine storm as well as promoting the regeneration of alveolar tissue, attributed to the intrinsic cytokines and growth factor present in the secretome. The preliminary studies have demonstrated the safety and efficacy of MSCs and exosomes in mitigating symptoms associated with COVID-19. Thus, they can be used on compassionate basis, owing to their ability to endogenously repair and decrease the inflammatory reactions involved in the morbidity and mortality of COVID-19. However, more preclinical and clinical studies are warranted to understand their mechanism of action and further establish their safety and efficacy.
\end{abstract}

Keywords Mesenchymal stem cells · Exosomes · COVID-19 · Cytokine storm · Acute respiratory distress syndrome

\section{Introduction}

The novel corona virus disease (COVID-19) has grown to be a global public-health emergency since the first case was detected in Wuhan, China, in December 2019. The novel corona virus or SARS-CoV-2 as named by the International Committee on Taxonomy of Viruses has over 14 million

Sachin Kadam

kadamsachin@gmail.com

Ashim Gupta

ashim6786@gmail.com

Shivaji Kashte

kashtesb@gmail.com

Manu Gupta

manu6771@yahoo.co.in

Hugo C. Rodriguez

hcrodrig2112@gmail.com

Shraddha Singh Gautam

shraddha.parab@advancells.com

1 Future Biologics, Lawrenceville, GA, USA confirmed cases worldwide and has claimed over 600,000 lives [1]. After 6 months from the first COVID19 diagnosis, we neither have an effective antiviral medication nor a vaccine available to deal with this emergency. Once infected, a patient mainly relies on their immunity to resist SARSCoV-2, with supportive treatment given if complications occur [2]. It has been confirmed that the first step in the

2 BioIntegrate, Lawrenceville, GA, USA

3 South Texas Orthopaedic Research Institute, Laredo, TX, USA

4 Veterans in Pain, Los Angeles, CA, USA

5 Department of Stem Cell and Regenerative Medicine, Centre for Interdisciplinary Research, D. Y. Patil Education Society (Institution Deemed To Be University), Kolhapur 416006, India

6 School of Osteopathic Medicine, University of the Incarnate Word, San Antonio, TX, USA

7 Advancells Group, Noida, A-102, Sector 5, Noida, Uttar Pradesh 201301, India 
SARS-CoV-2 pathogenesis is specific recognition of the angiotensin I converting enzyme 2 receptor (ACE2) by the viral spike protein [3], leading to an immune system overreaction causing damage to the body. The immune system overreaction in COVID-19 patients is associated with production of large amounts of inflammatory factors, causing a cytokine storm including an overproduction of immune cells like effector T cells and natural killer cells $[4,5]$.

Intensive Care Unit (ICU) admission rates are just over $5 \%$ from confirmed COVID-19 diagnosis [6], and more than half of these ICU patients showed higher plasma levels of granulocyte colony-stimulating factor (GCSF) and tumor necrosis factor alpha (TNF- $\alpha$ ). Increased GCSF and TNF- $\alpha$ have been found to produce a cytokine storm, leading to acute respiratory distress syndrome (ARDS) [3]. COVID-19 infected patients showed high circulating levels of proinflammatory cytokines including GCSF, IP10, MCP1, MIP1A and TNF- $\alpha$ levels, and the levels were higher in patients admitted to the ICU. As cytokine storm is the cause behind extensive lung damage that even leads to death, eliminating the cytokine storm and supressing superinflammatroy immunological response coupled with repair and regeneration of the lung tissue can be an effective treatment modality for COVID-19 [7]. Current treatments and clinical interventions for COVID-19 associated acute lung injuries include respiratory support (non-invasive ventilation and mechanical ventilation), antipyretic and non-specific antiviral drugs, corticosteroids, and immunoregulation [3]. With very high fatality rate seen among the critically ill ICU patient population that are unresponsive to the aforementioned treatment modalities, new approaches are required to mitigate the symptoms associated with COVID-19 infection [6, 8].

Cell-based therapies strive to treat or prevent injury and disease by naturally repairing, restoring, and/or regenerating damaged or diseased organs and tissues $[9,10]$. This field has exploded in recent years to meet the needs of patients with both complex and common medical problems [7]. Some cell-based therapies aim to slow or stop degenerative or pathophysiologic processes that ultimately present themselves as symptomatic conditions [7]. Other regenerative therapies activate the body's endogenous repair system by influencing the behaviour of somatic and progenitor cells to stop degenerating and start regenerating $[7,11]$. In the case of pneumonia, acute lung injury (ALI) [12], acute respiratory distress syndrome (ARDS) $[13,14]$ and sepsis studies investigating therapy using mesenchymal stem cells (MSCs) have demonstrated safety and some positive effects on these conditions [11]. A recent study conducted in Beijing You' an Hospital, Capital Medical University, China used MSCs in patients with COVID-19 pneumonia. The results demonstrated that symptoms such as fever, shortness of breath and low oxygen saturation disappeared and or improved 2-4 days' post treatment [3].
Several institutes worldwide are putting their best efforts to come up with a novel therapeutic option with good clinical safety and efficacy to help patients affected by COVID19. An early first attempt in China utilizing mesenchymal stem cells demonstrated potential to improve symptoms associated with COVID-19 [3]. Other studies also showed efficacy of intravenous administration of MSCs in boosting body's immune response against COVID-19 associated infection [3]. The mechanism of action was ascribed to the accumulation of intravenously administered MSCs into the lungs and help build up regenerative cells locally, which can potentially protect the epithelial cells of the lungs, and prevent lung damage [3]. The efficacy of MSCs can be attributed to their trans-differentiation; secretion of growth factors, cytokines and extracellular vesicles including exosomes and associated paracrine effect; and mitochondrial transfer $[15,16]$.

In addition to the stem cell therapy, a cell-free approach like the use of exosomes and associated bioactive molecules are leading the way to treat several pathologies. The exosomes produced by MSCs possess hypoimmunogenic properties are enclosed in a lipid bilayer making them extremely stable and are able to migrate to the target organ of damage instead of merely accumulating via blood flow. These extracellular vesicles are identified to be the primary factors responsible for paracrine effects detected in all types of stem cells and for the transfer of genetic material from stem cells to the tissue-specific cell that needs regeneration [17].

In search of a ultimate viable solution to the harrowing COVID-19, when scientists around the world are working on a number of vaccines and treatments for COVID-19, mesenchymal stem cells and their exosomes are emerging as a promising therapeutic option without any major adverse effects. The combinatorial strategy of antiviral drugs along with immunomodulatory, tissue protective and healing potential of stem cells and their exosomes may reduce the severity of the COVID-19. A call for an urgent development on the MSCs and their exosome-based therapeutics specifically targeted towards ARDS to ensure the health and survival of human being is strongly recommended.

\section{Mesenchymal stem cells and immunomodulation}

The therapeutic potential of MSCs due to their regenerative properties is well investigated in various degenerative and inflammatory disorders. MSCs are immunoprivileged cells, due to the low expression of class II Major Histocompatibilty Complex (MHC-II) and co-stimulatory molecules in their cell surface [18]. MSCs are known to hold an immunoregulatory capacity and elicit immunosuppressive effects by inhibiting T-cell proliferation to alloantigens and 
mitogens and prevent the development of cytotoxic T-cells [19] by interfering with various immune response pathways by means of direct cell-to-cell interactions and soluble factor secretion [18].

Profound immunomodulatory effects of MSCs on T and B cells and natural killer (NK) cells are mediated by regulatory $\mathrm{T}$ cells. MSCs are able to suppress in vitro T-cell proliferation induced by cellular or non-specific mitogenic stimuli through the secretion of various soluble factors that include TGF- $\beta$, HCF, PCE- 2 , IDO, HLA-G5 and NO. The effect of these suppressive factors is upregulated by pre-sensitization of MSCs with TNF- $\alpha$, and IFN- $\gamma$. MSCs polarizes T cells toward a regulatory phenotype that serve to decrease IL-6 and TNF- $\alpha$, which is an important mechanism by which MSCs dampen inflammation [20]. In addition, pretreatment of MSCs with IFN- $\gamma$ has been shown to also suppress B cells [11]. MSCs with the help of dendritic cells (DC) or monocytes are found to produce anti-inflammatory cytokines such as IL-10 that downregulates the human leucocyte antigen (HLA) class I [11] and exert protective effects including direct regeneration and secretion of multiple paracrine factors such as antibacterial peptides [20]. Interleukin-10 (IL10) plays an essential role in maintaining the immunomodulatory property of regulatory DC. While, regulatory DC play an important role in controlling immune homeostasis and can possess an immunosuppressive ability to induce specific immune tolerance and dampen $\mathrm{Th} 2$ type inflammation [3], MSCs have the ability to induce mature dendritic cells (DC) into novel Jagged-2 dependent regulatory dendritic cell population $[3,21]$. All these interactions with different dendritic cells lead to a shift of the immune system from a Th1 response toward an anti-inflammatory Th2 response [3].

\section{Mesenchymal stem cell therapy for COVID-19}

Cell-based therapy have gained a status as a promising therapeutic field, to cure incurable diseases like diabetes, cardiovascular diseases, neurodegenerative diseases, muscular degenerative disorders, cancers [10], liver injuries [9], hematopoietic and immune system disorders, metabolic disorders [7], graft-versus-host disease, sepsis and ARDS [20] due to their immunomodulation properties. Since the first observation of pluripotent undifferentiated cells resistance to murine polyomavirus infection [22], many researchers have explored the same against a variety of viral infections like Human Immunodeficiency Virus-1 (HIV1) and Human Immunodeficiency Virus-2 (HIV2) [23], hepatitis B virusrelated acute-on-chronic liver failure (HBV-ACLF) [7], Myxoma virus [24], Retrovirus [25], Cytomegalovirus [26], etc. with reference to the embryonic and adult stem cells. It was observed that the intrinsic expression of interferon stimulating genes (ISG) makes pluripotent and multipotent stem cells resistant to viral infections [27, 28]. It was further observed that ISG of stem cells gets this defence mechanism from interferon-induced transmembrane (IFITM) family of proteins [27, 28]. Although, stem cell therapy is not a method to eradicate or cure SARS-CoV-2, there are few evidence-based studies that support the concept that infected patients may be more likely to combat and survive the infection $[2,20]$. This can be attributed to the rejuvenation and regeneration properties of stem cells such as their ability to reduce inflammation, secrete cell protective substances, transfer mitochondria, decrease cell death, anti-oxidative effects, and improve overall immune function [29]. In addition, there is direct evidence that stem cells protect against the influenza virus (A/H5N1) infection, by helping reverse lung injury [29]. Owing to their anti-inflammatory, immunomodulatroy and homing properties as well as regenerative potential, MSCs have attracted the attention of many scientists as a cell based therapy for the treatment for COVID-19 [7].

Leng et al. conducted a clinical trial pilot study (ChiCTR2000029990) using human umbilical cord-derived MSCs (hUCMSCs) in seven COVID-19 patients for 14 days. Post-treatment, population of $\mathrm{CD} 14^{+} \mathrm{CD} 11 \mathrm{c}^{+} \mathrm{CD} 11 \mathrm{~b}^{\mathrm{Mid}}$ regDCs dramatically increased. The levels of TNF- $\alpha$ decreased significantly, while levels of IL-10 increased in the MSC treatment group compared to the placebo control group. The gene expression profile showed that MSCs were $\mathrm{ACE}^{-}$and TMPRSS2- ${ }^{-}$suggesting MSCs were free from COVID-19 infection. This study concluded that the intravenous transplantation of MSCs was safe and effective for treating patients with COVID-19 pneumonia, especially patients whose condition was critically severe. These results can be attributed to immunomodulatory role of MSCs in reversing the lymphocytes subsets [3].

In another single case study, intravenous allogenic hUCMSCs infused in a 65-year-old female critically ill of COVID-19 revealed that the pneumonia was greatly relieved and the patient recovered from ICU after 8 days. No hUCMSC-related side effects were observed. After first infusion, the serum bilirubin, C-reactive protein (CRP) and aspartate aminotransferase (AST)/ alanine aminotransferase (ALT) were gradually reduced. After 3 days, second administration was done and the white blood cells (WBCs) and neutrophil count decreased to the normal level, and the lymphocyte count increased to the normal level. Administration of hUCMSCs led to reduction in inflammation and recovery of antiviral immune cells and organs. In addition, it may have led to homing of hUCMSCs to repair the injured tissues and neutralize the inflammatory cytokines such as G-CSF and IL-6 by expression of their receptors [16]. It indicates that MSCs therapy might be an ideal choice to treat critically ill COVID-19 patients. 


\section{Mesenchymal stem cells' role in COVID-19 associated complications}

COVID-19 is associated with a number of complications with ARDS being the leading cause of the deaths. ARDS is known to be associated with protein-rich pulmonary edema and acute respiratory failure, characterized by acute inflammation and injury to the lungs and epithelia [13]. The primary treatment of COVID-19 remains supportive, involving lung protective ventilation and fluid conservation. ARDS is an important cause of morbidity and mortality with no definitive therapy [30]. The pathophysiology of ARDS involves an imbalance between proinflammatory and anti-inflammatory mediators. Therefore, regulation of those mediators, specifically cytokines, has been targeted as a potential therapeutic approach [31]. There are several studies that have demonstrated the therapeutic potential of MSCs for patients suffering with ARDS because of their specific immunomodulatory properties [31]. MSCs have been reported to treat impaired alveolar fluid clearance, decrease the lung permeability, combat infection and regulate inflammation in patients suffering from ARDS [31]. MSCs also have the ability to secrete a variety of soluble paracrine factors, including key antiinflammatory cytokines such as interleukin-10 (IL-10) and IL-1 receptor antagonist (IL-1RA) [31].

A preclinical study showed that human bone marrowderived mesenchymal stem cells (BMMSCs) were activated with the serum obtained from patients suffering from ARDS. These activated MSCs were more efficient in reducing lung inflammation compared to untreated MSCs. It resulted in increased expression of IL-10 and IL-1RA, which was associated with enhancement of their protective capacity by reduction of the lung injury score, development of pulmonary edema and accumulation of bronchoalveolar lavage inflammatory cells and cytokines [30]. In another study, BMMSCs were preconditioned with serum obtained from patients suffering with ARDS and activated. These activated MSCs secreted higher levels IL-1RA and IL-10, dampened the secretion of proinflammatory cytokines, exhibited upregulation of toll-like receptor 4 (TLR-4) and vascular endothelial growth factor (VEGF) genes, and triggered a strong immunomodulatory response via higher secretion of prostaglandin E2 (PGE2). When these activated allogenic MSCs were administered in ARDS porcine models, it suppressed proinflammatory cytokine levels and promoted secretion of anti-inflammatory mediators [31]. Thus, activation of allogenic MSCs through incubation in an environment previously exposed to MSCs may induce stronger immunomodulatroy effects in patients compared with infusion of nonactivated MSCs [31].

Additionally, clinical trials such as Stem cells for ARDS Treatment (START) trial, a multicentre, open label, dose escalation, phase I clinical trial (NCT01775774), utilized a single intravenous dose of bone marrow-derived mesenchymal stem cells (BMMSCs) which was given to nine moderate to severe ARDS patients. The results demonstrated no prespecified infusion-associated events or treatment-related adverse events. MSC infusion was well tolerated in all patients [14]. Furthermore, in a double blinded, multicentre randomized phase 2a trial (NCT02097641), one intravenous dose of BMMSCs was studied. Among 1038 patients screened, 60 were eligible and received treatment. The MSC dose was safe in patients with moderate to severe ARDS. There was no infusion-related haemodynamic or respiratory adverse events. It was concluded that larger trials will be needed to test further efficacy of MSCs for ARDS [13].

ARDS, lung failure and fulminant pneumonia are major symptoms associated with H7N9 infection. In a single-centre and open-label clinical trial (NTC0209544; ChiCTROCC-15006355), MSCs were transplanted into H7N9induced ARDS patients. In this clinical trial, 44 patients with H7N9-induced ARDS were included as control group without MSCs transplantation. The treatment group included 17 patients with H7N9-induced ARDS with allogenic menstrual blood-derived MSC transplantation. Results showed that the treatment group had a significantly lower mortality rate compared to the control group. The study also reported that MSC transplantation did not result in harmful effects in human body within the 5-years follow-up period. MSC transplantation significantly improved survival rate of H7N9-induced ARDS patients in both preclinical and clinical studies [32]. Considering that H7N9 and coronavirus share similar complications such as ARDS and corresponding multi-organ failure, MSC-based therapy could be a potential alternative for mitigating COVID-19 [32].

Another known and safe source of MSCs is Adipose tissue. Adipose-derived stem cells (ASCs) expresses a large amount of anti-inflammatory properties and can be obtained from a minimally invasive aspiration procedure [33]. ASCs secret factors that induce proliferation of vascular endothelial cells and angiogenesis that include VEGF and PDGF $[34,35]$. Along with their immunosuppressive activity due to the secretion of TGF-1, HGF and INF-y, they can potentially improve the pulmonary function of COVID-19 patients [36]. Recently, there was a phase I, single-centre, doubleblinded, placebo-controlled clinical trial (NCT01902082) that assessed the safety of ASCs in the treatment of ARDS [37]. The trial concluded that ASCs were safe with minimally adverse events and had potential to improve oxygenation in patients with moderate ARDS [37]. The results from this study are promising; however, more studies will be required to establish safety and efficacy of ASCs to treat patients infected with ARDS and COVID-19. 


\section{Mesenchymal stem cells and acute liver injury or acute liver failure}

Both SARS-COV-2 and the Middle East respiratory syndrome coronavirus have been known to induce acute liver injury (ALI). Studies have shown that patients with COVID19 ALI have an incidence rate as high as $11 \%$. ALI induced by viruses can progress rapidly to acute liver failure (ALF) [38]. ALI involves the infiltration of immune cells such as $\mathrm{T}$ cells, B cells and natural killer cells (NK). Immunosuppressive treatments and decreased inflammation reportedly promote repair after ALI [38]. MSCs have shown a ray of hope in repairing and regenerating liver tissues and in treatment of liver diseases [9].

A preclinical study demonstrated that ALI was significantly alleviated and survival rate of mice was improved post-treatment with MSCs. The results indicated that the MSCs modulated the hepatic immune system in terms of the distribution of immune cell subsets and phenotype of single cells. During the injury phase, MSCs exhibited a systemic response by reducing the number of Ly6 $\mathrm{C}^{\text {low }}$ $\mathrm{CD}^{+} \mathrm{T}^{\mathrm{RM}}$ cells, conventional NK cells*, and $\operatorname{IgM}^{+} \mathrm{IgD}^{+}$ $\mathrm{B}$ cells and increasing the number of immunosuppressive monocyte-derived macrophages. During recovery phase, MSCs promoted the retention of $\mathrm{Ly} 6 \mathrm{C}^{\text {low }} \mathrm{CD} 8^{+} \mathrm{T}^{\mathrm{RM}}$ cells and maintained activity of immunosuppressive monocytederived macrophages [38]. Another study demonstrated that the efficacy of MSC-based therapy is enhanced with lipid conjugated heparin coating; and the human adipose derived stem cells (hADSCs) delivered to the damaged liver resulted in significantly improved recovery from ALF in a mouse model. Results from this study showed that the intravenous administration of hADSCs lowered the elevated serum levels of aspartate transaminase (AST) and alanine transaminase (ALT). A significantly increased level of human hepatic growth factor (hHGF), a representative secretome from hADSCs, significantly reduced the levels of macrophage and CYP2E1 [39].

In another preclinical study, Bone marrow-derived mesenchymal stem cells (BMMSCs) were transfused in ALF rat models. The results demonstrated that BMMSCs inhibited neutrophil infiltration, oxidative activity and hepatocyte apoptosis. The BMMSCs also ameliorated ALF by increasing the expression of heme oxygenase (HO-1) [40]. HO-1 is known to possess the ability to reduce polymorphonuclear neutrophils (PMNs) infiltration and function, and thus, can play a vital anti-inflammatory and anti-apoptic role [40].

Another preclinical study utilizing transplantation of hUCMSCs in an ALI mice model, demonstrated the ability of these cells in decreasing the levels of hepatocellular necrosis and lobular neutrophilic infiltration, leading to significant hepatoprotective effects. No adverse effects, systemic toxicity or neoplastic finding related to hUCMSCs transplantation was observed [12]. In a similar study, intravenous administration of MSCs in an intoxicated and burn mice model resulted in less cellularity, limited apoptosis, and slight reduction in the proinflammatory cytokineinterleukin-6 (IL-6) and the neutrophil chemokine-KC (CXCL1) levels in the lung tissue. MSC treatment had more dramatic anti-inflammatory effects on systemic and hepatic inflammation. In summary, the results of this study indicated that MSC treatment can diminish systemic inflammation, lessen hepatic damage, and decrease liver and lung apoptosis and inflammation [41].

\section{Exosomes: The biological messengers of tissue repair}

Exosomes are the tiny packets filled with cellular proteins and nucleic acid materials (e.g. mRNA \& miRNA) released by stem cells [42]. Literature have demonstrated powerful regenerative potential of exosomes, ranging from immunemodulatory properties, anti-inflammatory properties, etc. [43]. Although exosomes have been discovered almost 30 years ago, it is only recently that scientists have generated immense interest for being one of the most promising, acellular alternatives to cellular therapeutics, due to their demonstrated aptitude [43]. Exosomes were first identified to be secreted from nanovesicles of sheep reticulocytes, at the time of their maturation; the experiment was performed in the year 1980, soon after which, exosomes were studied rapidly, as the secretions of all the cells including immune cells, like B and T lymphocytes and are found to be necessary for cellular communications [44]. Typically, exosomes are of the size of 40-150 nm and are found to be secreted through inward budding of the endosomes. Once released by the cells, they get entry into another cellular microenvironment, by fusing to the plasma membrane [45].

Studies have further indicated that increased cellular interaction, through cell to cell communication, is one of the most unique functions of exosomes [45]. Other than that, disposal of unwanted proteins, transfer of genetic material, elicitation of the immune response, reduction in inflammation, etc. are also identified to be some of the functional attributes of exosomes, depending upon the cell of origin $[44,45]$.

\section{Role of exosomes in COVID 19 infections}

Majority of the studies focusing on MSC-derived exosomes have demonstrated regenerative potential, immune-modulatory functions, anti-inflammatory effects, similar to their 
parents, i.e. Mesenchymal stem cells [44, 45]. In preclinical set up, MSC-derived exosomes have demonstrated aptitude as an acellular alternative to cell-based therapy, against Acute Respiratory Distress Syndrome (ARDS) [46]. These studies have further confirmed that post-exosomal infusion, the associated cytokine storm and pro-inflammatory signalling biomolecules were considerably reduced that were primarily responsible for ARDS pathogenesis [46]. Further analysis confirmed that the exosomes also increased the level of anti-inflammatory signalling mediators that can reduce the severity of the lung injury through increase permeability and functional aspects of alveolar epithelium $[47,48]$,

as a result of which, the exchange of oxygen-rich air is easily facilitated. Further deep diving into the same, the ability of exosomes to transfer mitochondria to alveolar cells further increased their survival rate, and thus, facilitated cellular regeneration. These effects have paved the way towards the therapeutic use of this novel acellular alternative [47]. Beyond their effects in preclinical model of acute lung disorders, MSC-derived exosomes were also found to be responsible for direct inhibition of viral multiplication $[47,49]$. With several studies investigating the bio-distribution of this cellular cargo in preclinical setup, it has been quite evident that these exosomes have the potential to alter a variety of different pathways to facilitate active cellular communication. The intrinsic component of the exosomes, miRNAs, are reportedly found to be the key component that is responsible for many physiological processes, like development, epigenetic alterations, immune regulations, etc.[50]. By using near IR dyes, several studies have figured out different techniques to track in-vivo biodistribution of exosomes upon systemic delivery in different animal models [50, 51].

Several studies have confirmed their reachability to different organs, like in intra-cerebral haemorrhagic rat models, exosomes could reach to the brain upon the intravenous administration [52]. Intravenous administration of exosomes in a mouse model with acute kidney injury shows their accumulation in the kidneys, further confirming exosomes strong paracrine pathways for instant reachability to the site of injury [53].

Multiple studies have demonstrated that miRNAs secreted by exosomes are very crucial for accelerated lung recovery, particularly in patients suffering from viral infections like influenza, hypoxia-induced pulmonary hypertension, ventricular induced lung injury, etc. Wang et al. observed and studied active regulation of miRNAs during early and late-stage repair of lung damage in the mouse model. This study further indicated that certain miRNAs like miR-290, miR-21, let-7 and miR-200 played a major role in lung regeneration, immune-regulation, and immune-modulation
[47]. Alipoor et al. presented strong experimental evidence that stem cell-derived exosomes can deactivate the signalling pathways associated with hypoxia that can also facilitate reduced hypertension and inflammation, specifically evident in the respiratory disorders [54]. Beyond their effects in a preclinical model of acute lung disorders, MSC-derived exosomes are also found to be responsible for direct inhibition of viral multiplication. Studies have confirmed that MSC-derived exosomes secrete miRNA, which acts as a silencing complex and further alters the expression of the cellular receptors through epigenetic changes that help in blocking the entry of many RNA viruses like influenza, hepatitis $\mathrm{C}$ and also Coronavirus $[47,55]$. In a pig model of influenza, intra-tracheal administration of MSC-derived exosomes, $12 \mathrm{hrs}$ post-infection, significantly reduced virus shredding [55].

\section{On-going clinical trials}

Several pre-clinical and clinical studies have explored the potential of MSCs and exosomes for treating COVID-19 including management of associated cytokine storm. Though the results are promising, the limited literature still warrants more studies to establish safety and efficacy of MSCs and exosomes to treat and manage symptoms associated with COVID-19 infection. Eventually, multi-center, controlled, randomized trials will be needed to adequately assess the future of MSCs as well as exosomes in the treatment of COVID-19. As of July 16, 2020, there are 55 on-going studies related to use of stem cells (Table 1) and 3 on-going studies related to use of exosomes (Table 2) registered on ClinicalTrials.gov.

\section{Conclusion}

COVID-19 is currently one of the biggest socio-economic and public health dangers that we have seen. With the hope of vaccine that will eradicate the viral infection still in the distant future, there is an urgent need for treatments that are not only efficacious but safe. Preliminary studies have demonstrated the safety and efficacy of MSCs and exosomes in mitigating symptoms associated with COVID-19. They can be used on compassionate basis, owing to their ability to endogenously repair and decrease the inflammatory reactions involved in the morbidity and mortality of COVID19 , but more preclinical and clinical studies are required to understand their mechanism of action and establish their safety and efficacy. 


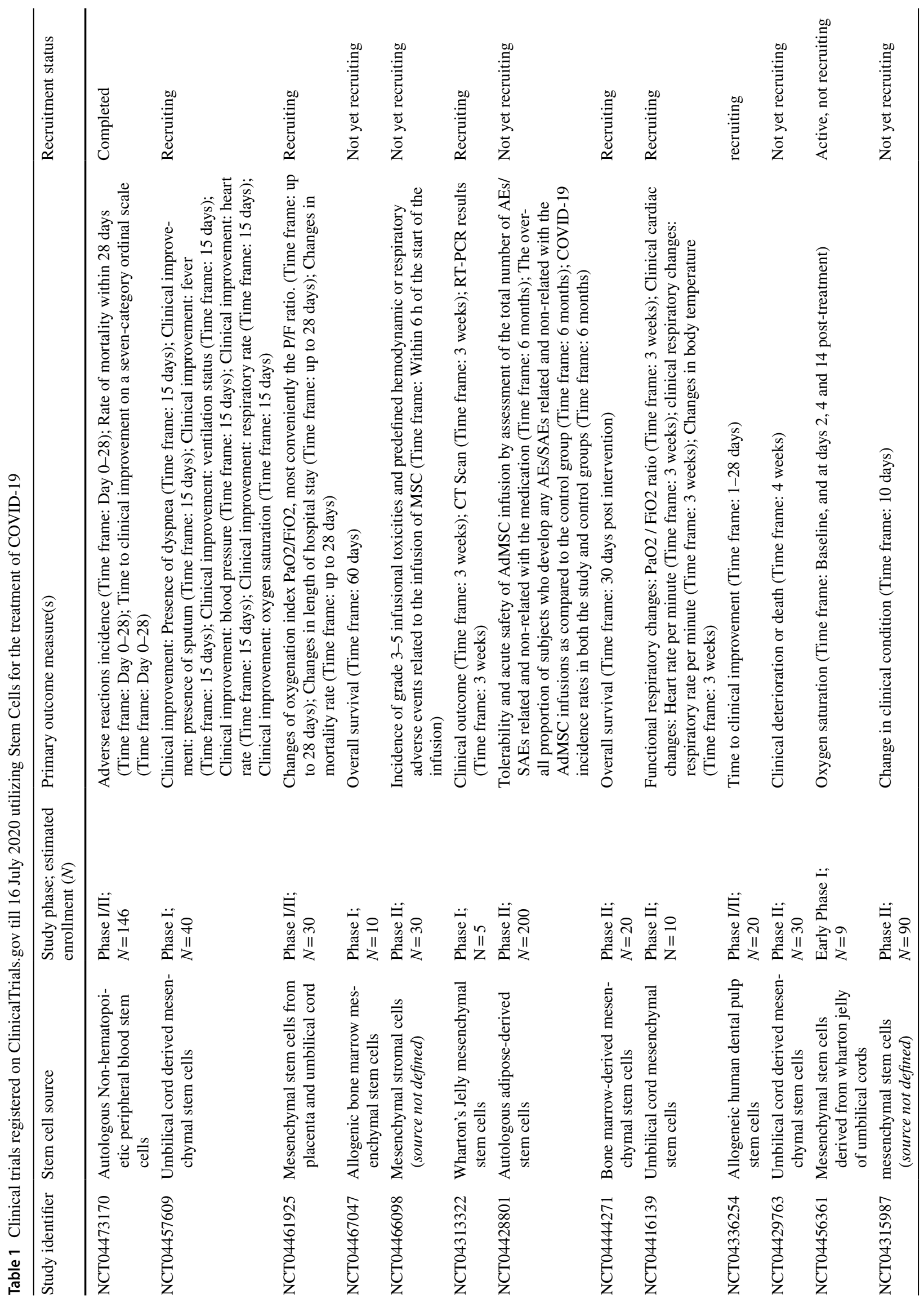




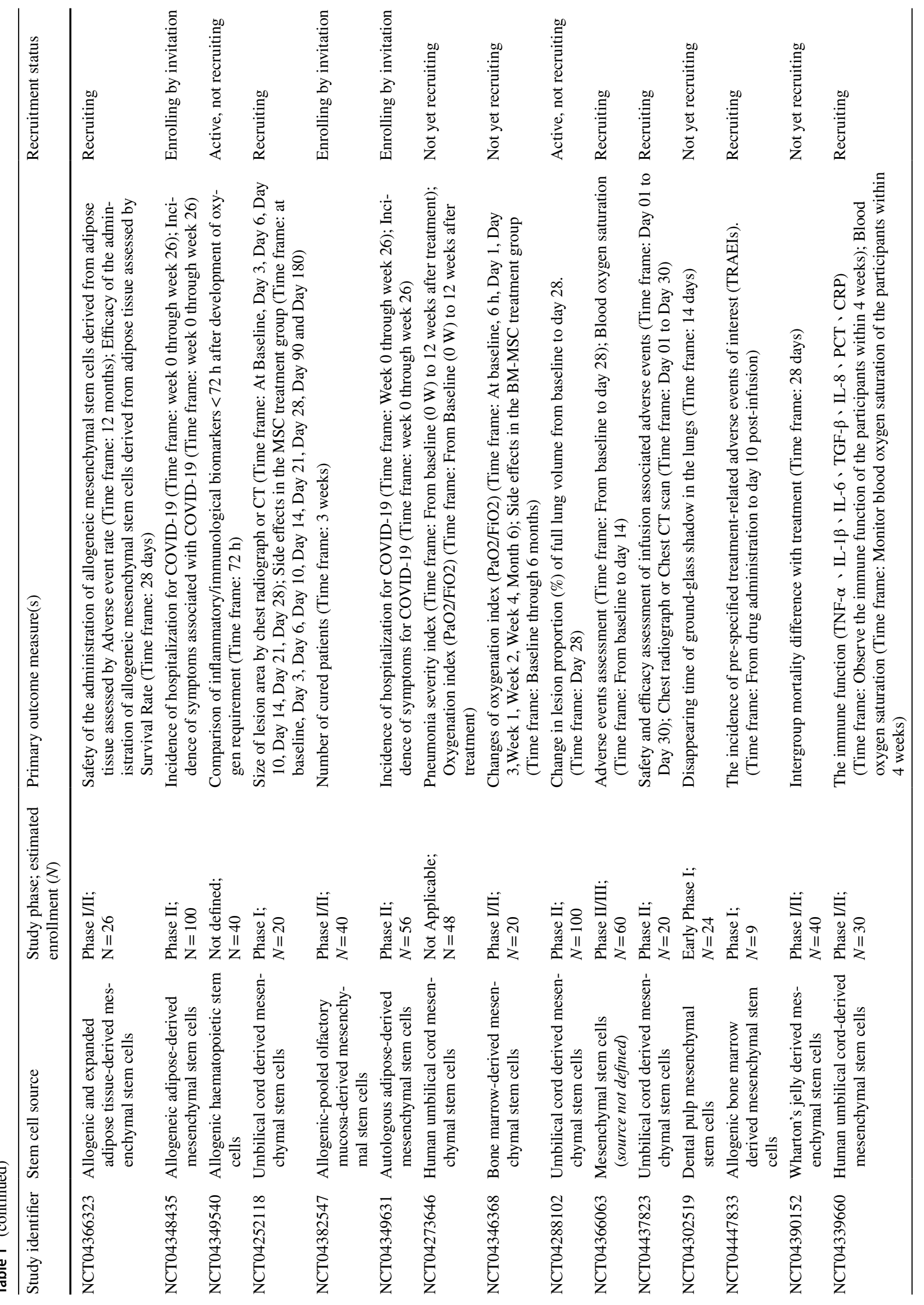




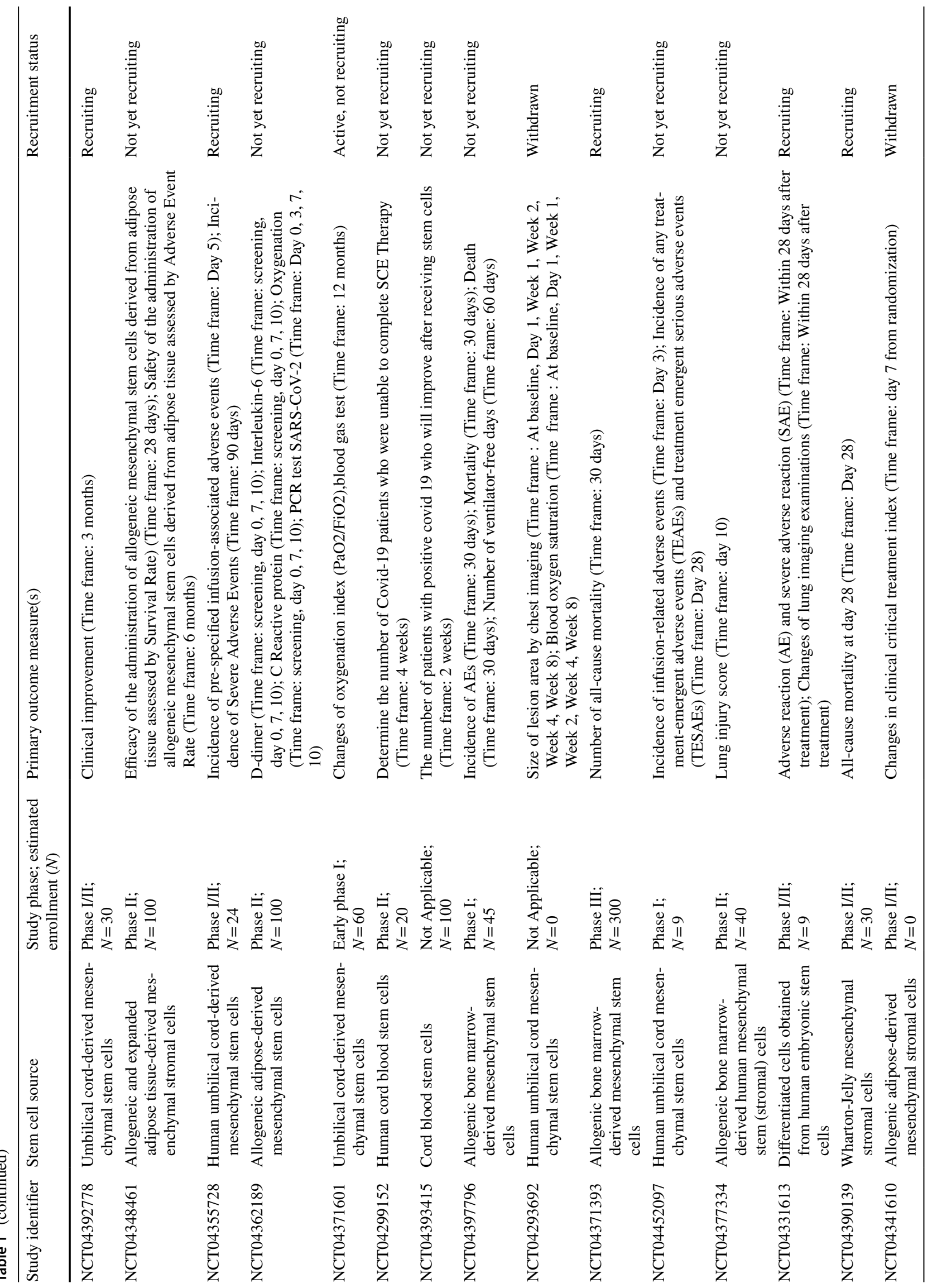




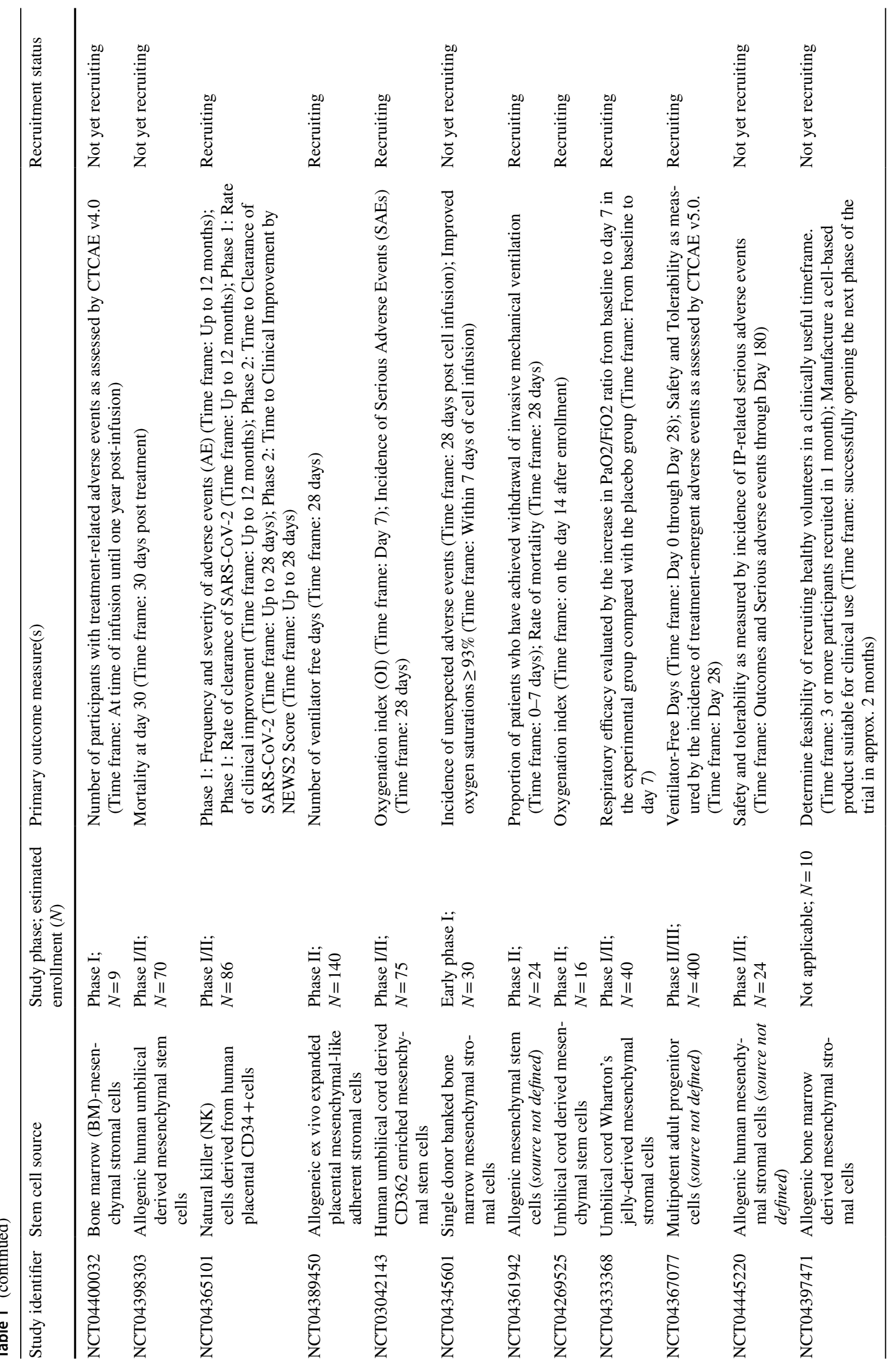


Table 2 Clinical trials registered on ClinicalTrials.gov till 16 July 2020 utilizing Exosomes for the treatment of COVID-19

\begin{tabular}{|c|c|c|c|c|}
\hline Study identifier & Exosome source & $\begin{array}{l}\text { Study Phase; esti- } \\
\text { mated enrollment } \\
\text { (N) }\end{array}$ & Primary outcome measure(s) & Recruitment status \\
\hline NCT04389385 & COVID-19 specific $\mathrm{T}$ cell & $\begin{array}{l}\text { Phase I; } \\
N=60\end{array}$ & $\begin{array}{l}\text { Adverse and severe adverse reaction (Time frame: } \\
28 \text { days); efficacy assessment (Time frame: } 28 \text { days); } \\
\text { rate of recovery without mechanical ventilator (Time } \\
\text { frame: } 28 \text { days) }\end{array}$ & Active, not recruiting \\
\hline NCT04384445 & Human amniotic fluid & $\begin{array}{l}\text { Phase I/II; } \\
N=20\end{array}$ & $\begin{array}{l}\text { Incidence of any infusion associated adverse events } \\
\text { (Time frame: } 60 \text { days); incidence of severe adverse } \\
\text { events (Time frame: } 60 \text { days) }\end{array}$ & Not yet recruiting \\
\hline NCT04276987 & $\begin{array}{l}\text { Allogenic adipose mesen- } \\
\text { chymal stem cells }\end{array}$ & $\begin{array}{l}\text { Phase I; } \\
N=30\end{array}$ & $\begin{array}{l}\text { Adverse reaction and severe adverse reaction (Time } \\
\text { frame: up to } 28 \text { days); time to clinical improvement } \\
\text { (Time frame: up to } 28 \text { days) }\end{array}$ & Not yet recruiting \\
\hline
\end{tabular}

\section{Compliance with ethical standards}

Conflict of interest The authors declare that they have no conflict of interest.

\section{References}

1. COVID-19 Dashboard by the Center for Systems Science and Engineering (CSSE) at Johns Hopkins University (JHU) [Internet]. [cited 2020 Jul 1]. Available from: https://coronavirus.jhu. edu/map.html

2. Zhao RC. Stem cell - based therapy for coronavirus disease 2019. Stem Cells Dev. 2020;29:679-81.

3. Leng Z, Zhu R, Hou W, Feng Y, Yang Y, Han Q, et al. Transplantation of ACE2 - Mesenchymal Stem Cells Improves the Outcome of Patients with COVID-19 Pneumonia. J Aging Dis. 2020;11:216-28.

4. Mangalmurti N, Hunter CA. Primer cytokine storms: understanding COVID-19. Immunity. 2020;53:19-25.

5. Hossein G, Zohreh M, Hedayat J, Zahra S. The possible pathophysiology mechanism of cytokine storm in elderly adults with COVID - 19 infection: the contribution of " inlame - aging". Inflamm Res. 2020;11:1-15.

6. Murthy S, Gomersall CD, Fowler RA. Care for Critically Ill Patients With COVID-19. J Am Med Assoc. 2020;323:1499-500.

7. Sleem A, Saleh F. Mesenchymal stem cells in the fight against viruses: face to face with the invisible enemy. Curr Res Transl Med. 2020. https://doi.org/10.1016/j.retram.2020.04.003.

8. Richardson S, Hirsch JS, Narasimhan M, Crawford JM, Mcginn T, Davidson KW. Presenting characteristics, comorbidities, and outcomes among 5700 patients hospitalized with COVID-19 in the New York City area. JAMA. 2020;323:2052-9.

9. Kashte S, Maras JS, Kadam S. Bioinspired engineering for liver tissue regeneration and development of bioartificial liver: A review. Crit Rev Biomed Eng. 2018;46:413-27.

10. Kashte S, Kadam S. Stem cell therapy: a hope business or a magic wand? Br Biomed Bull. 2014;2:677-94.

11. Rawat S Gupta S, Mohanty S. Mesenchymal stem cells modulate the immune system in developing therapeutic interventions. In: Tyagi RK, Bisen PS, editors. Immune Response Act. IntechOpen; 2019. pp. 1-25.
12. Yun J, Ahn JH, Kwon E, Kim S, Kim H, Kim WH, et al. Human umbilical cord-derived mesenchymal stem cells in acute liver injury: hepatoprotective efficacy, subchronic toxicity, tumorigenicity, and biodistribution. Regul Toxicol Pharmacol. 2016;81:437-7.

13. Matthay MA, Calfee CS, Zhuo H, Thompson BT, Wilson JG, Levitt JE, et al. Treatment with allogeneic mesenchymal stromal cells for moderate to severe acute respiratory distress syndrome (START study): a randomised phase 2 a safety trial. Lancet Respir. 2018;2600:1-9.

14. Wilson JG, Liu KD, Zhuo H, Caballero L, Mcmillan M, Fang X, et al. Mesenchymal stem (stromal) cells for treatment of ARDS: a phase 1 clinical trial. Lancet Respir. 2015;3:24-322.

15. Paliwal S, Chaudhuri R, Agrawal A, Mohanty S. Regenerative abilities of mesenchymal stem cells through mitochondrial transfer. J. Biomed. Sci. 2018;25:1-12.

16. Liang B, Chen J, Li T, Wu H, Yang W, Li Y, et al. Clinical remission of a critically ill COVID-19 patient treated by human umbilical cord mesenchymal stem cells. Medicine. 2020;99(31):e21429.

17. Syndrome RD. COVID-19 does not lead to a "Typical”" acute respiratory distress syndrome. Am J Respir Crit Care Med. 2020;201:1299-300.

18. De MMP, Pascual CY, Aller MA, Arias J. Immunosuppressive properties of mesenchymal stem cells: advances and applications. Curr Mol Med. 2012;12:574-91.

19. Le BK, Ringde O. Immunomodulation by mesenchymal stem cells and clinical experience. J Intern Med. 2007;262:509-25.

20. Zhang J, Xie B, Hashimoto K. Current status of potential therapeutic candidates for the COVID-19 crisis. Brain Behav Immun. 2020;87:0-15.

21. Zhang B, Liu R, Shi D, Liu X, Chen Y, Dou X, et al. Mesenchymal stem cells induce mature dendritic cells into a novel Jagged-2 dependent regulatory dendritic cell population. Blood J. Am. Soc. Hematol. 2009;113:46-57.

22. Vitro CI. Neoplastic differentiation: interaction of simian virus 40 and polyoma virus with murine teratocarcinoma. J Cell Physiol. 1975;85:179-87.

23. Weichold FF, Zella D, Barabitskaja O, Maciejewski JP, Dunn DE, Sloand EM, Young NS. Neither human immunodeficiency virus-1 (HIV-1) nor HIV-2 infects most-primitive human hematopoietic stem cells as assessed in long-term bone marrow cultures. Blood J. Am. Soc. Hematol. 1998;91:907-15.

24. Villa NY, Bais S, Chan WM, Meacham AMYM, Wise E, Rahman MM, et al. Ex vivo virotherapy with myxoma virus does 
not impair hematopoietic stem and progenitor cells. Cytotherapy. 2016;18:465-80.

25. Wolf D, Goff SP. Embryonic stem cells use ZFP809 to silence retroviral DNAs. Nature. 2009;458:1201-4.

26. Belzile J, Stark TJ, Yeo GW, Spector H. Human cytomegalovirus infection of human embryonic stem cell- derived primitive neural stem cells is restricted at several steps but leads to the persistence of viral DNA. J Virol. 2014;88:4021-39.

27. Bailey CC, Zhong G, Huang IC, Farzan M. IFITM-family proteins: the cell's first line of antiviral defense charles. Annu Rev Virol. 2014;1:261-83.

28. Huang Y, An X, Rosenberg BR, Rice CM, Wu X, Loan V, et al. Stem cells intrinsic immunity shapes viral resistance of stem cells. Cell. 2018;172(423-424):e25.

29. Chan MCW, Kuok DIT, Leung CYH, Hui KPY, Valkenburg SA, Lau EHY, et al. Human mesenchymal stromal cells reduce influenza A H5N1-associated acute lung injury in vitro and in vivo. Proceed National Acad Sci. 2016;113:3621-6.

30. Bustos ML, Huleihel L, Meyer EM, Donnenberg AD, Donnenberg VS, Sciurba JD, Mroz L, McVerry BJ, Ellis BM, Kaminski N, Rojas M. Activation of human mesenchymal stem cells impacts their therapeutic abilities in lung injury by increasing interleukin ( IL ) -10 and IL-1RN levels. Stem Cells Transl. Med. 2013;2:884-95.

31. Xu AL, Rodriguez LA, Walker KP III, Mohammadipoor A, Kamucheka RM, Cancio LC, Batchinsky AI, Antebi B. Mesenchymal stem cells reconditioned in their own serum exhibit augmented therapeutic properties in the setting of acute respiratory distress syndrome. Stem Cells Trans Med. 2019;8(10):1092-106.

32. Chen J, Hu C, Chen L, Tang L, Zhu Y, Xu X, et al. Clinical study of mesenchymal stem cell treating acute respiratory distress syndrome induced by epidemic Inluenza A (H7N9) infection: A hint for COVID-19 treatment. Engineering. 2020. https://doi. org/10.1016/j.eng.2020.02.006.

33. Mazini L, Rochette L, Amine M, Malka G. Regenerative capacity of adipose derived stem cells (adscs), comparison with mesenchymal stem cells (MSCs). Int J Mol Sci. 2019;20:2530.

34. Gentile P, Garcovich S. Concise review: adipose-derived stem cells (ASCs) and adipocyte-secreted exosomal microRNA (A-SE$\mathrm{miR}$ ) modulate cancer growth and promote wound repair. J Clin Med. 2019;8(6):855.

35. Gentile P, Casella D, Palma E, Calabrese C. Engineered fat graft enhanced with adipose-derived stromal vascular fraction cells for regenerative medicine: clinical, histological and instrumental evaluation in breast reconstruction. J Clin Med. 2019;8:504.

36. Gentile P, Sterodimas A. Adipose-derived stromal stem cells (ASCs) as a new regenerative immediate therapy combating coronavirus (COVID-19) -induced pneumonia. Expert Opin. Biol. Ther. 2020;00:1-6.

37. Zheng G, Huang L, Tong H, Shu Q, Hu Y, Ge M, et al. Treatment of acute respiratory distress syndrome with allogeneic adiposederived mesenchymal stem cells: a randomized, placebo-controlled pilot study. Respir Res. 2014;15:1-10.

38. Liu J, Feng B, Xu Y, Zhu J, Feng X, Chen W, et al. Immunomodulatory effect of mesenchymal stem cells in chemical-induced liver injury: a high-dimensional analysis. Stem Cell Res Therapy. 2019;10:1-13.

39. Hwang Y, Kim JC, Tae G. Significantly enhanced recovery of acute liver failure by liver targeted delivery of stem cells via heparin functionalization. Biomater. 2019;209:67-78.
40. Zhang Z, Zhu W, Ren H, Zhao X, Wang S, Ma H, et al. Mesenchymal stem cells increase expression of heme oxygenase-1 leading to anti-inflammatory activity in treatment of acute liver failure. Stem Cell Res Therapy. 2017;8:1-13.

41. Curtis BJ, Shults JA, Boe DM, Ramirez L, Kovacs EJ. Mesenchymal stem cell treatment attenuates liver and lung inflammation after ethanol intoxication and burn injury. Alcohol. 2018;80:139-48.

42. Huang-doran I, Zhang C, Vidal-puig A. Extracellular vesicles: novel mediators of cell communication in metabolic disease. Trends Endocrinol Metab. 2016;28:3-18.

43. Toh WEIS, Zhang BIN, Lai RC, Lim SAIK. Immune regulatory targets ofmesenchymal stromal cell exosomes/ small extracellular vesicles in tissue regeneration. Cytotherapy. 2018;20:1419-26.

44. Harding CV, Heuser JE, Stahl PD. Exosomes: Looking back three decades and into the future. J Cell Biol. 2013;200:367-71.

45. Zhang Y, Liu Y, Liu H, Tang WH. Exosomes: biogenesis, biologic function and clinical potential. Cell Biosci BioMed Central. 2019;9:9-19.

46. Abraham A, Krasnodembskaya A. Mesenchymal stem cell-derived extracellular vesicles for the treatment of acute respiratory distress syndrome: Concise review. Stem Cells Transl Med. 2019;9:28-38.

47. Wang M, Yuan Q, Xie L. Review article mesenchymal stem cellbased immunomodulation: properties and clinical application. Stem Cells Int. 2018;2018:12.

48. Worthington EN, Hagood JS. Therapeutic use of extracellular vesicles for acute and chronic lung disease. Int J Mol Sci. 2020;21:2318.

49. Progenitor T. Exosomal microRNAs derived from umbilical mesenchymal stem cells inhibit hepatitis $\mathrm{C}$ virus infection. Stem Cells Transl Med. 2016;5:1190-203.

50. Id LM, Sadovsky Y. The biology of extracellular vesicles: The known unknowns. PLoS Biol. 2019;17:e3000363.

51. Yi YW, Lee JH, Kim S, Pack C, Ha DH, Park SR, et al. Advances in analysis of biodistribution of exosomes by molecular imaging. Int J Mol Sci. 2020;21:665.

52. Xiong Y, Ph D, Mahmood A, Chopp M. Emerging potential of exosomes for treatment of traumatic brain injury mechanisms underlying cell therapy for TBI MSC-derived exosome as a novel therapy for TBI. Neural Regen Res. 2020;12:19-22.

53. Kholia S, Beatriz M, Sanchez H, Cedrino M, Papadimitriou E, Tapparo M, et al. Mesenchymal stem cell derived extracellular vesicles ameliorate kidney injury in aristolochic acid nephropathy. Front cell Dev Biol. 2020;8:1-17.

54. Alipoor SD, Mortaz E. Exosomes in respiratory disease. In: Edelstein L, Smythies J, Quesenberry P, Noble D, editors. Exosomes. Academic Press; 2020. p. 383-414.

55. Khatri M, Richardson LA, Meulia T. Mesenchymal stem cell-derived extracellular vesicles attenuate influenza virusinduced acute lung injury in a pig model. Stem Cell Res Ther. 2018;9:1-13.

Publisher's Note Springer Nature remains neutral with regard to jurisdictional claims in published maps and institutional affiliations. 